\title{
3D Compressed Spectrum Mapping with Sampling Locations Optimization in Spectrum-Heterogeneous Environment
}

\author{
Feng Shen, Student Member, IEEE, Zheng Wang, Member, IEEE, \\ Guoru Ding, Senior Member, IEEE, Kezhi Li, and Qihui Wu, Senior Member, IEEE
}

\begin{abstract}
Spectrum mapping has emerged as an important problem in wireless communications, which generates a spectrum map for the spectrum resource analysis and management. Given the constrained transceiver volume and the limited energy consumption, how to effectively reconstruct the spectrum situation by the limited sampling data is a pressing challenge for spectrum mapping. In this paper, by exploiting the sparse nature of spectrum situation, we firstly attempt to solve the three-dimensional (3D) compressed spectrum mapping problem in the way of compressed sensing. Then, we develop a quadrature and right-triangular $(\mathrm{QR})$ pivoting based measurement matrix optimization algorithm. By iteratively selecting new dominant sampling locations, it promotes the recovery accuracy compared to random measurement. After that, we propose a 3D spatial subspace based orthogonal matching pursuit (OMP) algorithm to recover spectrum situation for 3D compressed spectrum mapping. Finally, simulations are presented to show the comparisons in terms of localization, source signal strength recovery, recovery success rate and situation recovery. Results show our proposed 3D spectrum mapping scheme not only effectively reduces the sampling number, but also achieves a high level of spectrum mapping accuracy.
\end{abstract}

Index Terms-Spectrum mapping, compressed sensing, spectrum-heterogeneity, OMP algorithm

\section{INTRODUCTION}

W ITH the proliferation of various wireless devices and the increasingly crowded spectrum environment, how to efficiently utilize spectrum resources has received much attention [1]-[3]. As reported by the Federal Communications Commission (FCC), most of licensed bands allocated to for

This work is supported by the National Natural Science Foundation of China (No. 61827801, No. 61801216, No. 61871398, No. 61931011, No. 61631020), the Natural Science Foundation for Distinguished Young Scholars of Jiangsu Province (No. BK20190030), the Natural Science Foundation of Jiangsu Province (No. BK20180420), the open research fund of Key Laboratory of Dynamic Cognitive System of Electromagnetic Spectrum Space (Nanjing Univ. Aeronaut. Astronaut.), Ministry of Industry and Information Technology, Nanjing, 211106, China and No. U20B2038. (Corresponding author: Zheng Wang and Qihui Wu)

F. Shen and Q. Wu are with Key Laboratory of Dynamic Cognitive System of Electro-magnetic Spectrum Space (Nanjing Univ. Aeronaut. Astronaut.), Ministry of Industry and Information Technology Nanjing 211106, China (email: sfjx_nuaa@163.com,wuqihui2014@sina.com).

Z. Wang is with School of Information Science and Engineering, Southeast University, Nanjing 210096, China (e-mail:z.wang@ieee.org). He is also with with College of Electronic and Information Engineering, Nanjing University of Aeronautics and Astronautics (NUAA), Nanjing, China.

G. Ding is with the College of Communications Engineering, Army Engineering University, Nanjing 210007, China (e-mail: dr.guoru.ding@ieee.org).

$\mathrm{K}$. Li is with Institute of Health Information, University College London, London NW1 2DA, UK (e-mail: Ken.li@ucl.ac.uk). example broadcasting TVs or analogue cellular telephony are often highly underutilized [4], [5]. Consequently, to solve such a widespread dilemma in most current wireless systems with fixed spectrum access, the revolutionary spectrum sharing technology, cognitive radio (CR) [6]-[9] comes out. It has overlay, underlay and interweave modes in resource allocations and access strategies [2], [10], [11]. The key task in cognitive radio is to accurately determine the spectrum situation for dynamic spectrum access of radio frequency (RF) devices, which is called spectrum situational awareness in the DARPA RadioMap program [12]. Here spectrum situation refers to the current state of the electromagnetic environment, including busy or idle spectrum state, spectrum signal strength, spectrum modulation mode, and spectrum access protocol, etc.

Spectrum mapping has become a new interest in wireless communication, which aims at establishing a spectrum map to effectively manage the spatial spectrum resources in spectrumheterogeneous environment. Therein the spectrum situational awareness results are projected to the geographic locations one by one, which indicates the signal strength at any location in space. With the 3D spatial spectrum map, the RF devices can instantly access the idle spectrum in both underlay/interweave modes and avoid conflicts where it is busy. This undoubtedly and greatly helps spectrum sharing and interference management, and thereby improves the spectrum utilization [13]. Due to constrained signal acquisition costs and limited hardware computation of RF devices, the target in CR spectrum mapping is accurate and robust spectrum reconstruction with limited sampling points.

With respect to $3 \mathrm{D}$ spectrum mapping, there are three main challenges. Firstly, most of existing works focus on 2D spectrum mapping. In [14], the authors propose a prototype of a radio environment map (REM) which aims at storing and reasoning the spectrum data obtained from heterogeneous spectrum sensors. This is a basic guiding work of constructing a heterogeneous radio environment map for spectrum management. In [15], the authors apply nonlinear support vector machine (SVM) to detect the boundary between the coverage area and the non-coverage area of the primary user, which is actually a prototype of a 2D binary spectrum map. In [16], support vector machine-type solvers are used to obtain power spectrum map, which also minimizes the bandwidth requirements for sensor measurements by introducing linear compression and quantization. Moreover, the authors in [17] propose a new power spectrum estimation algorithm based 
on generating adversarial networks (GANs) for detecting idle radio resources, which transforms the estimation task into an image reconstruction task through image color mapping. However, these 2D ground spectrum maps do not well match the practical 3D spectrum-heterogeneous environment. What's more, as discussed in [18], by leveraging the location flexibility of newly emerging RF devices such as unmanned aerial vehicle (UAV), it is able to explore more spatial spectrum opportunities. Specifically, when the RF device is at different heights, whether it can occupy the spectrum and how much impact it has on the primary user will be different. Therefore, due to more spatial freedom and greater spatial isolation between primary user and secondary users, the spectrum access opportunity which does not exist in 2D plane can be further exploited in 3D space. Therefore, the spectrum mapping along with the height dimension is worthy being considered, which leads to 3D spectrum mapping.

Secondly, 2D spectrum mapping is usually treated as solving a matrix completion problem. In [19]-[21], the authors formulate the spectrum situation of multiple frequency points with multiple time slots as an "image" and propose an idea of image inference to complete and predict the spectrum data. However, these works only deal with spectrum data in time-frequency dimension, the geographical location is not combined, nor is signal transmission mechanism taken consideration. What's more, when a column or row of the spectrum matrix is completely missing, it is rather difficult to recover the desired matrix [21]. For this reason, the technique of compressed sensing (CS) (also known as compressed sampling) is applied in this paper to recover the entire 3D spectrum situation map, which allows a high signal reconstruction performance even with a small number of measurements by taking advantage of the sparseness of the signal [22]-[25]. Common recovery algorithms include matching pursuits (MP) algorithm [26], orthogonal matching pursuit (OMP) algorithm [27] and so on.

Thirdly, most of the existing works employ random sampling over locations to return the sampling data for spectrum mapping. However, different from the traditional random sampling [28], [29], if the intrinsic features of the spatial spectrum situation could be fully exploited, it is possible to restore the 3D spectrum situation map with less data requirement. Studies in [30] explore optimized sensor placement based on a tailored library of features extracted from training data by means of QR factorization with column pivoting. In our prior work [31], region of interest (ROI) driven 3D spectrum sampling procedure is studied, which aims to obtain better ROI spectrum situation recovery with less energy cost. Nevertheless, the channel propagation characteristics for signal transmission is not taken into consideration. This is also one of the listed open issues for 3D spectrum mapping.

In a nutshell, most of existing studies focus on 2D ground spectrum mapping with spectrum data collected by random sampling. To the best of our knowledge, there are no reports on 3D spectrum mapping with optimized measurements in the spectrum-heterogeneous environment, which will be studied in this paper based on compressed sensing. The main contributions of this paper are summarized as follows:

- The 3D compressed spectrum mapping model is firstly formulated in the way of compressed sensing by exploiting the sparse nature of spectrum situation.

- A QR pivoting based 3D compressed spectrum mapping measurement matrix optimization algorithm is developed. The optimization successively executes a sparse dictionary preprocessing, selects new sampling points with the largest column 2-norms and applies Householder reflection. Comparisons confirm our solution outperforms random measurement by less spectrum mapping errors.

- An improved 3D spatial subspace based OMP algorithm for 3D compressed spectrum recovery is proposed. It solves the sparse signal representation of the 3D space and recovers the 3D spatial spectrum situation, which overcomes the ineffectiveness of the traditional OMP algorithm in compressed sensing. Performance comparison$\mathrm{s}$ show the superiority of our proposed 3D compressed spectrum mapping scheme.

The remainder of this paper is organized as follows. Section II introduces and formulates the 3D spectrum mapping model, and reviews the compressed sensing model. In Section III, the 3D compressed spectrum mapping model is proposed. Then, the details of 3D spectrum mapping scheme are presented in Section IV. Section V presents the simulation results and Section VI draws conclusions.

\section{PRELIMINARIES ON 3D SPECTRUM MAPPING AND COMPRESSED SENSING}

In this paper, we consider constructing a 3D spectrum map as shown in Fig. 1, in which spectrum signals from building facilities, vehicles, human being, etc. spread everywhere. As illustrated in Fig. 1(a), the entire area to be monitored is a 3D space which is spectrum-heterogeneous. The area with high spectrum energy (in red) indicates busy spectral occupancy, and the area with low spectrum energy (in yellow) indicates that the spectrum channel is relatively idle. The purpose of $3 \mathrm{D}$ spectrum mapping is to get a complete and accurate 3D spectrum situation map for efficient and reliable dynamic spectrum access management, in which we can obtain the spectrum occupancy status as well as spectrum channel quality of any position in the 3D space of interest.

\section{A. 3D Spectrum Mapping}

In order to solve the task of 3D spectrum mapping, we discretize the entire 3D space into small cubes as shown in Fig. 1(b). The color of each cube represents the signal strength of this position. Red indicates high signal strength and green indicates low strength. This constitutes a spectrum tensor $\mathcal{X} \in \mathbb{R}^{N_{1} \times N_{2} \times N_{3}}$ of the 3D space, where $N_{1}, N_{2}$, and $N_{3}$ represent the grids number of tensor $\mathcal{X}$ in $x, y, z$ dimensions, respectively.

In the process of spectrum mapping, it is supposed to perform spatial spectrum signal strength measurements in every cube. However, our goal is to operate spectrum sensing in as few cubes as possible and recover the energy values of all unsampled cubes to get the complete 3D spectrum map as shown in Fig. 2. If we sample every location, we will undoubtedly obtain the accurate spectrum map, however, it 


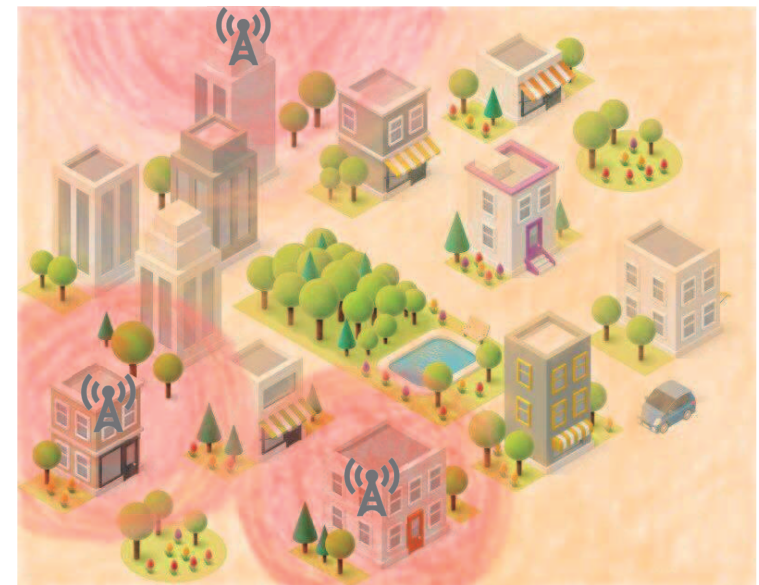

(a) An illustration of the spectrum situation for a city block scenario.

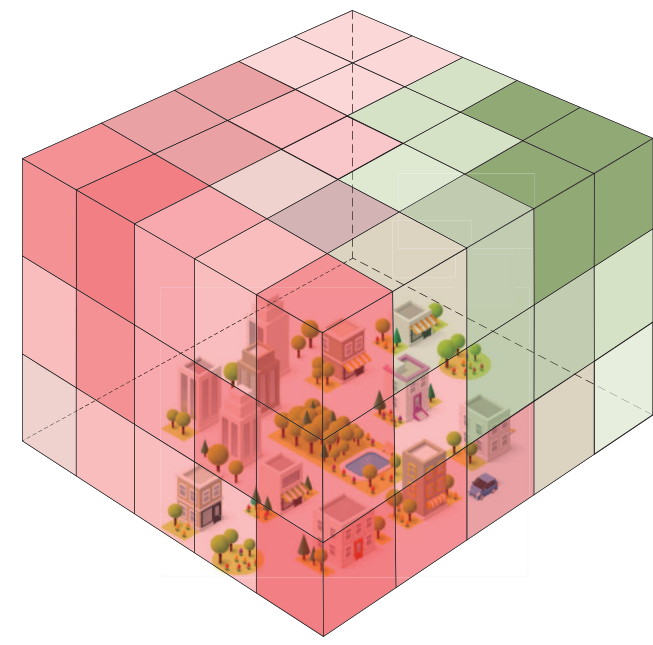

(b) A discretized three-dimensional spatial spectrum situation tensor of (a).

High Signal Strength

Low Signal Strength

((\&) Signal Source

Fig. 1. System Model. (a) illustrates the spectrum situation of a city block, different places have different spectral signal strengthes. (b) is a 3D tensor obtained by discretizing the 3D space in (a). Each cube has a situation value, the red means high signal strength, and the green represents low signal strength.

will suffer enormous resource consumption. Therefore, we have to adaptively search for suitable sampling positions to avoid blind sampling. By exploiting the spatial correlations among the sampling locations, it is possible to restore the spectrum situation through partial sampling. Of course, if the sampling ratio is too small, the spatial correlation among all the sampling points will be severely damaged, resulting in poor recovery accuracy. In addition, even with the same sampling ratio, different sampling locations combinations will lead to different recovery accuracies [31].

Thus, the objective is to obtain better spectrum situation recovery, subject to limited sampling ratio by optimizing the sampling locations as follows:

$$
\begin{gathered}
\Theta^{*}=\underset{\Theta}{\arg \min } W_{\mathcal{X}}, \\
\text { s.t. } r=\frac{N_{\Theta}}{N_{1} \times N_{2} \times N_{3}}, \\
W_{\mathcal{X}}=\frac{1}{N_{\mathcal{X}}} \sum_{i \in \mathcal{X}}\left(\frac{\left|\mathcal{T}_{\mathcal{X}_{R}^{i}}-\mathcal{T}_{\mathcal{X}^{i}}\right|}{\mathcal{T}_{\mathcal{X}^{i}}}\right)^{2},
\end{gathered}
$$

where $\Theta$ represents a set of sampling points, including the number of samples and the sampling positions. $W_{\mathcal{X}}$ is the Relative Mean Square Error (RMSE) of the target 3D space $\mathcal{X} . N_{\Theta}$ denotes the sampling number, and $r$ is the sampling ratio. In (C1-2), the $N_{\mathcal{X}}$ represents the total points number of $\mathcal{X} . \mathcal{T}_{\mathcal{X}^{i}}$ and $\mathcal{T}_{\mathcal{X}_{R}^{i}}$ represent the original and recovered spectral energy value at the $i$-th position, respectively. Eq. (1) is a function of $\boldsymbol{\Theta}$, which means given a sampling ratio $r$, a total of $\frac{\left(N_{1} \cdot N_{2} \cdot N_{3}\right) !}{\left(r \cdot N_{1} \cdot N_{2} \cdot N_{3}\right) !\left((1-r) \cdot N_{1} \cdot N_{2} \cdot N_{3}\right) !}$ deployment solutions can be found. This scale is too large to be solved by means of exhaustive search if $N_{\mathcal{X}}$ is big. Traditionally, for the spectrum mapping problem illustrated in Fig. 2, researchers usually treat it as a tensor recovery problem [19], or perform matrix completion by decomposing the tensor into a series of matrices to finally obtain the recovered tensor $\mathcal{X}_{R}$ [20], [21]. Therein, the objective is minimizing $\left\|\mathcal{T}_{\mathcal{X}_{R}}-\mathcal{T}_{\mathcal{X}}\right\|_{F}$. However, with a fixed sampling ratio, the sampling locations are selected in advance or randomly generated.

\section{B. Compressed Sensing}

In compressed sensing theory, it is assumed that the projection of the signal $\boldsymbol{x} \in \mathbb{R}^{n \times 1}$ on dictionary $\psi \in \mathbb{R}^{n \times l}$ is sparse, and the sparsity is $k\left(k=\|s\|_{0}\right.$ represents the nonzero number in signal $s \in \mathbb{R}^{l \times 1}, s$ is called sparse representation of the original signal $\boldsymbol{x}$ ). The observation vector $\boldsymbol{y} \in \mathbb{R}^{m \times 1}$ can be obtained by multiplying measurement matrix $\phi \in \mathbb{R}^{m \times n}$, that is, $\boldsymbol{y}=\boldsymbol{\phi} \boldsymbol{x}=\boldsymbol{\phi} \boldsymbol{\psi} \boldsymbol{s}$, where $\boldsymbol{\psi}$ is called sparse dictionary, and $\phi$ is called measurement matrix. Since $m \ll n$, reconstructing the original signal $\boldsymbol{x}$ through the observation $\boldsymbol{y}$ is an ill-condition process. With compressed sensing theory, this problem is transformed into the following optimization problem:

$$
\begin{gathered}
\boldsymbol{s}^{*}=\underset{\boldsymbol{s}}{\arg \min }\|\boldsymbol{s}\|_{0}, \\
\text { s.t. }\left\|\boldsymbol{y}-\boldsymbol{\phi} \boldsymbol{x}^{*}\right\|^{2} \leq \sigma^{2}, \\
\boldsymbol{x}^{*}=\boldsymbol{\psi} \boldsymbol{s}^{*} .
\end{gathered}
$$

The $\ell_{0}$ minimization problem in Eq. (2) is NP-hard so it is tough to solve but it is equivalent to the $\ell_{1}$ minimization problem as follows:

$$
\begin{gathered}
\boldsymbol{s}^{*}=\underset{\boldsymbol{s}}{\arg \min }\|\boldsymbol{s}\|_{1}, \\
\text { s.t. }\left\|\boldsymbol{y}-\boldsymbol{\phi} \boldsymbol{x}^{*}\right\|^{2} \leq \sigma^{2}, \\
\boldsymbol{x}^{*}=\boldsymbol{\psi} \boldsymbol{s}^{*} .
\end{gathered}
$$


Usually the problem in Eq. (3) is solved by using greedy pursuit algorithms such as orthogonal matching pursuit (OMP) [27].

\section{3D COMPRESSED SPECTRUM MAPPING MOdEL}

The difficulty of applying compressed sensing to 3D spectrum mapping lies in mining the inherent sparsity feature of 3D spectrum situation. Actually, the spectral energy strength $\mathcal{T}_{\mathcal{X}^{i}}$ for each location is generated by linear superposition of signals from all sources in the 3D space after path loss and is then added with noise. Compared with the total discretized points in the 3D space in Fig. 1, the number of signal sources in space turns out to be sparse in fact. Therefore, based on Section II, the 3D compressed spectrum mapping model is established as follows:

As shown in Fig. 3, the entire $N \times N \times N\left(N_{1}=\right.$ $N_{2}=N_{3}=N$ ) situation tensor $\mathcal{X}$ is first vectorized into $\boldsymbol{x} \in \mathbb{R}^{N^{3} \times 1}$. Then, the sparse representation of the spectrum situation is analyzed as:

$$
s_{\mathcal{X}^{i}}= \begin{cases}p_{\mathcal{X}^{i}}, & \text { if a sourse exists at point } \mathcal{X}^{i}, \\ 0, & \text { else, }\end{cases}
$$

where $p_{\mathcal{X}^{i}}$ is the transmit power of the signal at position $\mathcal{X}^{i}$. $k=\left\|\boldsymbol{s}_{\mathcal{X}}\right\|_{0}$ represents the sparseness of $\boldsymbol{s}_{\mathcal{X}}, s_{\mathcal{X}} \in \mathbb{R}^{N^{3} \times 1}$ is a sparse signal with exactly $k$ nonzero elements corresponding to $k$ signal sources in the 3D space.

According to the path loss of signal propagation model, the spectral signal strength at any point in $3 \mathrm{D}$ space can be obtained as:

$$
\boldsymbol{x}_{\mathcal{X}^{j}}=\sum_{l=1}^{N_{\mathcal{X}}} s_{\mathcal{X}^{l}} d_{j l}^{-\eta}
$$

where $\eta$ is path loss coefficient determined by the environment. $d_{j l}$ represents the Euclidean distance of radio signal propagation between point $\mathcal{X}^{j}$ and point $\mathcal{X}^{l}$, which is defined as:

$$
d_{j l}=\left\{\begin{array}{l}
\left\|\mathcal{X}^{j}-\mathcal{X}^{l}\right\|_{2}, \quad j \neq l, \\
1, \quad j=l
\end{array}\right.
$$

Compared to the compressed sensing model, we obtain the sparse dictionary $\boldsymbol{\psi}_{j l}^{3 D S M}=f\left(d_{j l}\right)=d_{j l}^{-\eta}$ for $3 \mathrm{D}$ spectrum mapping. Because of $d_{j l}=d_{l j}$, the sparse dictionary $\boldsymbol{\psi}^{3 D S M}$ is a symmetric matrix. Moreover, we let $\boldsymbol{x}_{\mathcal{X}}$ denote the spectral signal strength vector of all points, which is equivalent to $\mathcal{T}_{\mathcal{X}}$ in Eq. (1) of the spectrum mapping model.

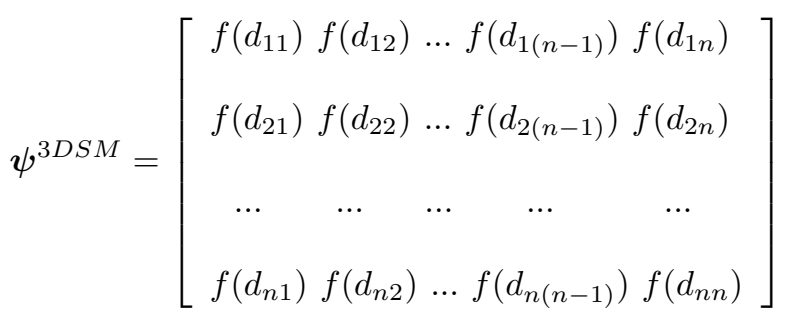

According to the obtained signal strength of each position in 3D space, we can perform spatial sampling operation by selecting $m$ sampling positions from all $n$ positions with

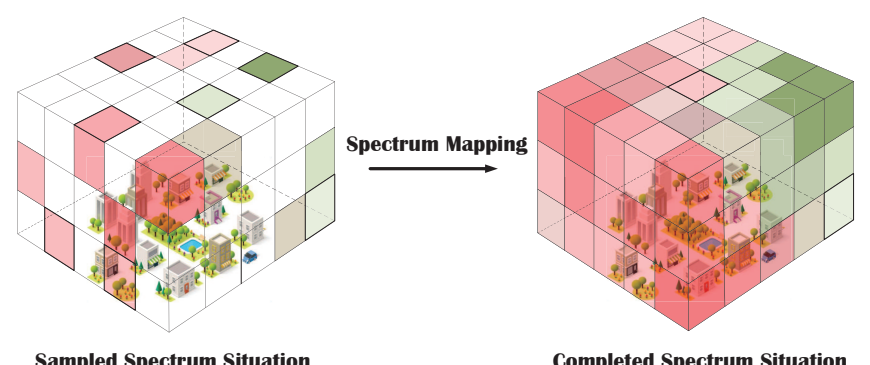

Fig. 2. Graphical illustration of 3D spectrum mapping. It aims at accurately recovering the situational values of all locations in the entire 3D space through a small number of sampling points.
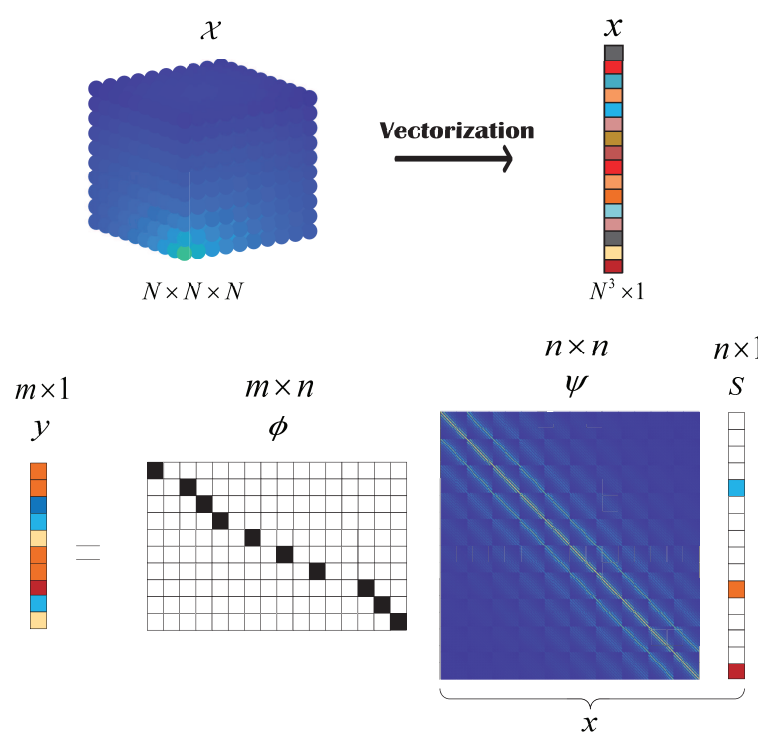

Fig. 3. Schematic illustration of 3D spectrum mapping process based on compressed sensing. The entire $N \times N \times N$ situation tensor $\mathcal{X}$ is first vectorized into $\boldsymbol{x}\left(\mathbb{R}^{N^{3} \times 1}\right)$. Then, according to Eq. (4) and (5), $\boldsymbol{x}$ can be expressed as the product of the sparse dictionary $\psi\left(\mathbb{R}^{n \times n}, n=N^{3}\right)$ and the sparse signal $s\left(\mathbb{R}^{n \times 1}\right)$. $\phi$ represents the sampling matrix, where the black and white squares represent 1 and 0 , respectively. $\boldsymbol{y}\left(\mathbb{R}^{m \times 1}\right)$ is the received signal. The goal of the entire mapping is to accurately recover the complete $\boldsymbol{x}$ with $\boldsymbol{y}$.

sampling ratio $m / n$ (according to Eq. (1) and (2), $n=N_{\mathcal{X}}$ ). Therefore, we have:

$$
\boldsymbol{y}^{3 D S M}=\boldsymbol{\phi}^{3 D S M} \boldsymbol{x}_{\mathcal{X}}+\boldsymbol{\varepsilon}=\boldsymbol{\phi}^{3 D S M} \boldsymbol{\psi}^{3 D S M} \boldsymbol{s}_{\mathcal{X}}+\boldsymbol{\varepsilon},
$$

where $\varepsilon$ denotes the Gaussian additive white noise with power spectral density $\sigma_{0}^{2}$. The measurement matrix $\phi^{3 D S M}$ satisfies

$$
\phi_{i j}^{3 D S M}=\left\{\begin{array}{l}
1, \text { point } \mathcal{X}^{j} \text { is the } i \text { th sampling location, } \\
0, \text { point } \mathcal{X}^{j} \text { is not sampled, }
\end{array}\right.
$$

where each row of $\phi^{3 D S M}$ has an element of 1 representing the sampling position. Therefore, the objective of 3D spectrum sensing is rewritten as:

$$
\begin{gathered}
\hat{\boldsymbol{s}}_{\mathcal{X}}=\underset{\boldsymbol{s}_{\mathcal{X}}}{\arg \min }\left\|\boldsymbol{s}_{\mathcal{X}}\right\|_{1}, \\
\text { s.t. }\left\|\boldsymbol{y}^{3 D S M}-\boldsymbol{\phi}^{3 D S M} \hat{\boldsymbol{x}}_{\mathcal{X}}\right\|^{2} \leq \sigma^{2}, \\
\hat{\boldsymbol{x}}_{\mathcal{X}}=\boldsymbol{\psi}^{3 D S M} \hat{\boldsymbol{s}}_{\mathcal{X}} .
\end{gathered}
$$


(a) $r=0.2$

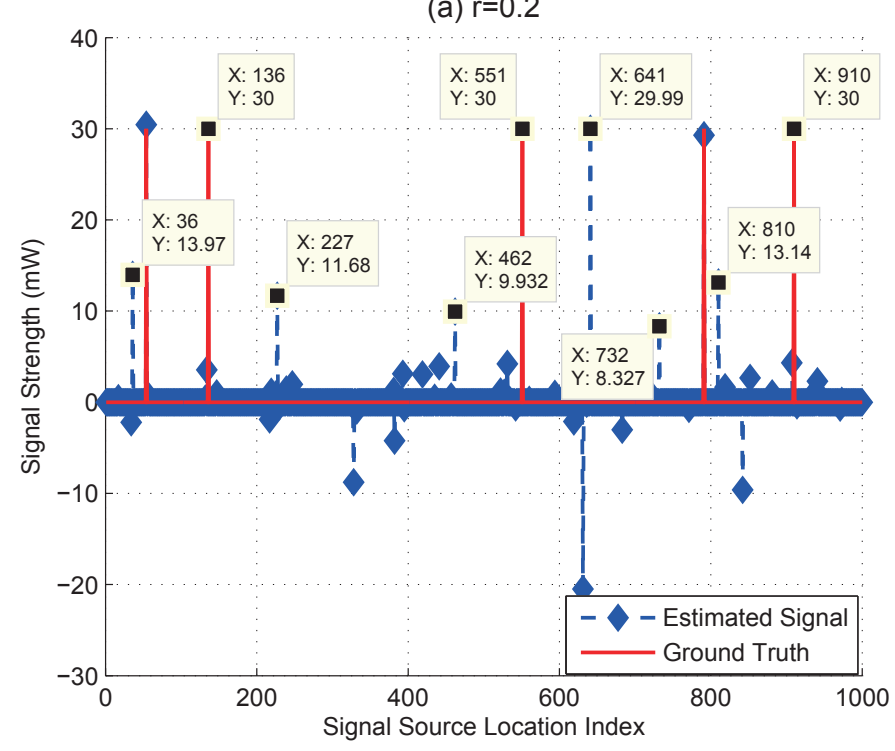

(b) $r=0.3$

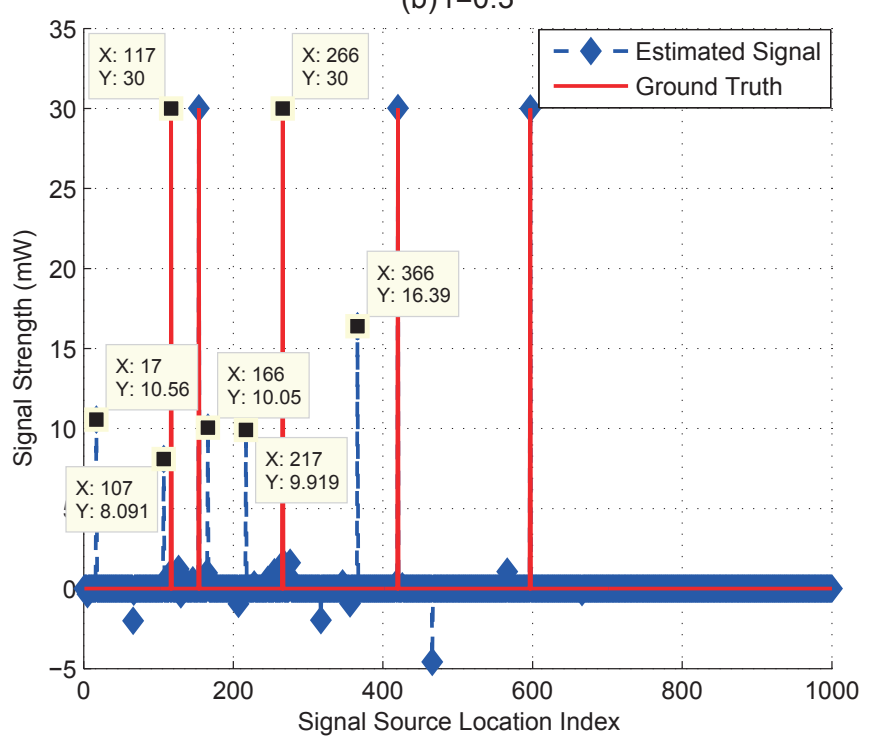

Fig. 4. Sparse signal $\boldsymbol{x}$ recovery performance with random sampling matrix $\phi$ and traditional OMP recovery algorithm. The sampling rates are 0.2 and 0.3 for (a) and (b) respectively. It is observed that the estimated signal source locations and signal strengthes are not accurate, however, the estimated locations are always adjacent to the real signal sources in the Cartesian coordinate.

Without considering the optimization of the sampling position$\mathrm{s}$, i.e., the measurement matrix, we adopt random sampling in three-dimensional space and perform signal recovery with OMP algorithm. The result is shown in Fig. 4. Clearly, the recovery algorithm based on traditional compressed sensing and no sampling position optimization has poor performance. It is unable to accurately restore the locations of all sources in the 3D spectrum map. However, it is observed that the positions of the restored signal sources are adjacent or close to the real signal sources in Cartesian coordinate. Therefore, it is necessary to propose a $3 \mathrm{D}$ compressed spectrum mapping scheme to coping with the difficulty in accurate $3 \mathrm{D}$ spectrum situation recovery.

\section{3D COMPRESSED SPECTRUM MAPPING SCHEME}

In this section, the proposed 3D compressed spectrum mapping scheme is introduced, which contains sampling positions (measurement matrix) optimization and 3D spectrum situation reconstruction algorithm.

\section{A. QR pivoting based measurement matrix optimization}

The QR decomposition factors an input matrix $G$ as the product of an orthogonal matrix $Q$ and a right triangular matrix $\boldsymbol{R}$, i.e., $\boldsymbol{G}=\boldsymbol{Q} \boldsymbol{R}$. Nevertheless, the above standard $\mathrm{QR}$ algorithm is not suitable for purposes like rank determination or low rank approximations. In [32], it is shown that a column permutation operation helps to find more representative columns during decomposition, which is named as QR decomposition with column pivoting. Therein, a permutation matrix $\boldsymbol{P}$ is required to rearrange columns into a more beneficial ordering which is represented as $\boldsymbol{G P}=\boldsymbol{Q R}$. Accordingly, a series of applications with QR pivoting have emerged, such as least-squares approximation, as well as various measurement selection tasks [33]-[36].

When solving Eq. (10), the invertibility of the product about $\phi^{3 D S M}$ and $\psi^{3 D S M}$ has a greater impact on the recovery of sparse signal $s_{\mathcal{X}}$. The invertibility of the matrix can be measured by the condition number, which is defined as the ratio of the maximum and minimum singular values of the matrix. What's more, the condition number of the system may be indirectly bounded by optimizing the spectral content of matrix using its determinant, trace or spectral radius [30].

The oversampled sensor placement problem is studied in [30], where the number of sensors exceeds the number of modes used in reconstruction. The key computational idea is the QR factorization with column pivoting which yields $\varpi$ sensors (pivots) that best sample the $\varpi$ basis modes of $\psi_{\varpi}$ (case $m=\varpi$ ):

$$
\boldsymbol{\psi}_{\varpi}^{T} \boldsymbol{C}^{T}=\boldsymbol{Q R},
$$

where the column permutation matrix $C$ actually represents the sensor placement matrix. Moreover, the measurement matrix is optimized by maximizing the matrix determinant [30]:

$$
\boldsymbol{\gamma}^{*}=\underset{\boldsymbol{\gamma},|\boldsymbol{\gamma}|=m}{\operatorname{argmax}}\left|\operatorname{det} \boldsymbol{M}_{\boldsymbol{\gamma}}\right|=\underset{\boldsymbol{\gamma},|\boldsymbol{\gamma}|=m}{\arg \max } \prod_{i}\left|\boldsymbol{\lambda}_{i}\left(\boldsymbol{M}_{\boldsymbol{\gamma}}\right)\right|,
$$

therein $\boldsymbol{M}_{\gamma}=\boldsymbol{C}_{\gamma} \boldsymbol{\psi}_{\varpi}$ and $\gamma$ represents the $m$ selected columns. $\lambda_{i}$ is the eigenvalue of $\boldsymbol{M}_{\boldsymbol{\gamma}}$. $\operatorname{det}(\cdot)$ denotes the operation of matrix determinant calculation. According to Eq. (11) and (12), we get

$$
\begin{aligned}
\left|\operatorname{det} \boldsymbol{M}_{\boldsymbol{\gamma}}\right| & =\left|\operatorname{det}\left(\boldsymbol{C}_{\boldsymbol{\gamma}} \boldsymbol{\psi}_{r}\right)\right|=|\operatorname{det}(\boldsymbol{Q R})| \\
& =|\operatorname{det} \boldsymbol{Q}||\operatorname{det} \boldsymbol{R}|=|\operatorname{det} \boldsymbol{R}| \\
& =\prod_{i}\left|a_{i i}\right| .
\end{aligned}
$$




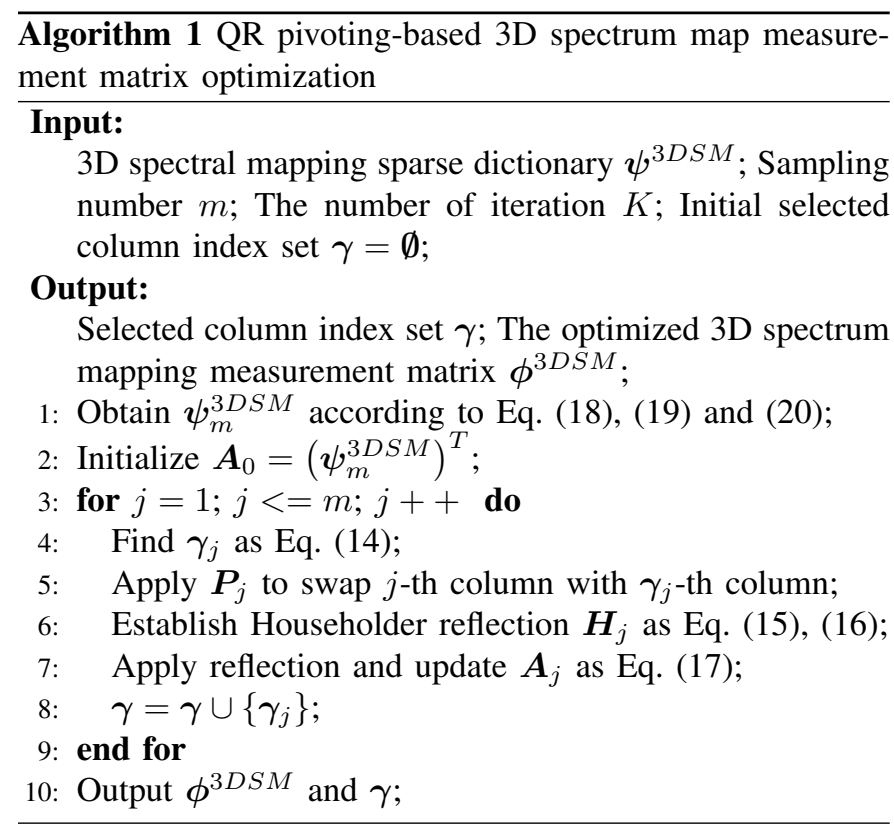

Therefore, the objective of maximizing the first $m$ eigenvalues of matrix $M_{\gamma}$ can be turned into maximizing the product of the first $m$ diagonal items of the matrix $\boldsymbol{R}$.

QR pivoting algorithm for the above optimization is an approximate greedy solution, which is known as submatrix volume maximization (here the matrix volume refers the absolute value of the determinant). At the $j$-th iteration, QR pivoting algorithm increments the submatrix volume by selecting new dominant column with maximum 2-norm:

$$
\gamma_{j}=\underset{j \notin\{1,2, \ldots, j-1\}}{\arg \max }\left\|\boldsymbol{a}_{j}\right\|_{2} .
$$

where $\boldsymbol{a}_{j}=\left(a_{j j}, a_{j+1 j}, \ldots, a_{n j}\right)^{T}$ is $j$-th column of $\boldsymbol{A}_{j-1}$.

Then, a Householder transformation is applied on the dominant column by

$$
\boldsymbol{H}_{j} \cdot \boldsymbol{a}_{\boldsymbol{\gamma}_{j}}=\left(\left|\boldsymbol{a}_{\boldsymbol{\gamma}_{j}}\right|, 0, \ldots, 0\right)^{T},
$$

where $\boldsymbol{H}_{j}=\boldsymbol{I}-2 \boldsymbol{\omega}_{j} \boldsymbol{\omega}_{j}^{T}, \boldsymbol{\omega}_{j}$ denotes the reflection vector:

$$
\omega_{j}=\frac{a_{\gamma_{j}}-\left|a_{\gamma_{j}}\right| e}{\left|a_{\gamma_{j}}-\right| a_{\gamma_{j}}|e|} .
$$

The $\boldsymbol{a}_{\gamma_{j}}$ denotes the column with the maximum 2-norm in the $j$-th iteration. $e$ is a unit vector with 1 at the first index and zeros elsewhere. $\boldsymbol{e}$ has the same length as $\boldsymbol{a}_{\boldsymbol{\gamma}_{j}}$.

Moreover, a permutation matrix $\boldsymbol{P}_{j}$ is introduced to swap $j$-th column with $\gamma_{j}$-th column. The Householder reflection contributions from every other column onto the pivot column are further subtracted by multiplying $\operatorname{diag}\left(\boldsymbol{I}_{j-1}, \boldsymbol{H}_{j}\right)$. Thus, the $\boldsymbol{A}_{j}$ is updated as

$$
\boldsymbol{A}_{j}=\operatorname{diag}\left(\boldsymbol{I}_{j-1}, \boldsymbol{H}_{j}\right) \cdot \boldsymbol{A}_{j-1} \cdot \boldsymbol{P}_{j} .
$$

This $\mathrm{QR}$ pivoting procedure is recomputed for each new column selection and gradually expands the submatrix volume by enforcing a diagonal dominance structure [37]. After $m$ iterations, we obtain the $m$ dominant sampling positions. If necessary, $n$ iterations are executed to get a total list of

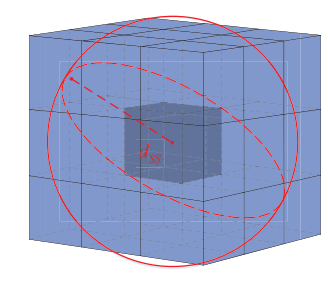

(a) An illustration of spatial subspace

All elements in the subspace

Spatial subspace region

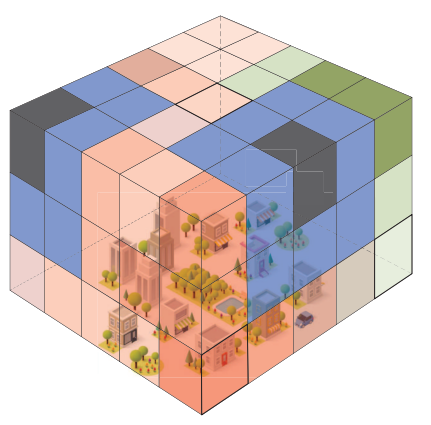

(b) Spatial subspaces in the 3D space

Center of the spatial subspace

$d_{S S} \quad$ Spatial subspace radius
Fig. 5. An illustration of the 3D spatial subspace. The red sphere is defined as a spatial subspace with a radius $d_{S S}$. All spatial cubes within the sphere form a set to solve the next dominant sampling location. (b) presents some examples of spatial subspaces.

$n$ pivots, with the first $m$ pivots optimized for spectrum reconstruction. The additional sampling locations may also be leveraged if available.

However, our sparse dictionary $\psi^{3 D S M}$ is a full-rank matrix as shown in Eq. (7), that is, $m<\operatorname{rank}\left(\psi^{3 D S M}\right)=n$. It can't be directly applied to the case of Eq. (11). Thus, we add a sparse dictionary preprocessing by executing singular value decomposition (SVD) on the sparse matrix $\psi^{3 D S M}$ in advance as Eq. (18), (19) and (20).

$$
\begin{gathered}
{\left[\boldsymbol{U}_{n \times n}, \boldsymbol{S}_{n \times n}, \boldsymbol{V}_{n \times n}\right]=\operatorname{svds}\left(\boldsymbol{\psi}^{3 D S M}, n\right),} \\
\boldsymbol{V}_{n \times m}=\boldsymbol{V}_{n \times n}(1: m,:), \\
\boldsymbol{\psi}_{m}^{3 D S M}=\boldsymbol{U}_{n \times n} \times \boldsymbol{S}_{n \times n} \times \boldsymbol{V}_{m \times n}^{T},
\end{gathered}
$$

where $\operatorname{svds}(\boldsymbol{M}, n)$ represents the SVD decomposition of matrix $M$ and obtains the largest $n$ eigenvalues and their corresponding eigenvectors. For the $n$ singular values of the original sparse dictionary, only the first $m$ items are kept. Based on the above preprocessing and QR pivoting algorithm, we can quickly get the optimized measurement matrix $\phi_{m}^{3 D S M}$, which is also the basis for the following spectrum mapping recovery stage.

The entire pseudo implementation code is summarized in Algorithm 1. Therein line 1 is sparse dictionary preprocessing. Line 3 to line 9 are the QR pivoting procedures. Specifically, it selects the pivot that has the maximum column 2-norms at each iteration and updates trailing column to remove the contribution of row $j$ according to the Householder transformation.

The time complexity of sparse dictionary preprocessing is $\mathcal{O}\left(n^{3}\right)$. The rest time complexity mainly focuses on line 4 and line 7 . For the $j$-th iteration, the complexity of line 4 is $\mathcal{O}((m-j-1)(n-j-1))$. Based on the matrix multiplication law, the computation cost of line 7 is $\mathcal{O}\left(m^{2} n+m n^{2}\right)$. Therefore, the total time complexity of Algorithm 1, denot- 
ed by $\left(\mathcal{C}_{1}\right)$, is

$$
\begin{aligned}
\mathcal{C}_{1} & =O\left(n^{3}\right)+O\left(\sum_{j=1}^{m}(m-j-1)(n-j-1)\right) \\
& +O\left(m\left(m^{2} n+m n^{2}\right)\right) \\
& =O\left(n^{3}+m^{2} n+m^{3} n+m^{2} n^{2}\right) .
\end{aligned}
$$

\section{B. Improved 3D Spatial Subspace Based OMP Algorithm For $3 D$ Compressed Spectrum Mapping Recovery}

In the traditional OMP algorithm, the target is to continuously select column index in the sensing matrix $\phi \psi$ which has the largest inner product with the iteratively updating residual. Due to the ineffective performance of the traditional OMP algorithm in 3D spectrum situation recovery as shown in Fig. 4, we propose an improved OMP algorithm based on 3D spatial subspace for efficient spectrum recovery as shown in Algorithm 2.

First, for the selection of each optimal column (line 2 in Algorithm 2), we design a spatial subspace as shown in Fig. 5(a). At the $t$-th iteration, the chosen column (sampling location) at the center of the 3D spatial subspace is

$$
\pi_{t}^{c}=\underset{i=1,2, \ldots, n}{\arg \max }\left|\left\langle\boldsymbol{q}_{t-1}, \boldsymbol{\delta}_{i}\right\rangle\right|,
$$

where $\boldsymbol{q}_{t-1}$ represents the residual and $\boldsymbol{\delta}_{i}$ is the $i$-th column of the sensing matrix $\Delta=\phi \psi$. Operation $\langle\cdot, \cdot\rangle$ means inner product calculation between two vectors.

Then, all columns within the spatial subspace radius $d_{S S}$ are found in Cartesian coordinate and denoted as $\boldsymbol{d}_{t}^{\text {set,xyz }}$ (line 3 to 4 in Algorithm 2) according to

$$
\left\|\mathcal{X}^{\pi_{t}^{i}}-\mathcal{X}^{\pi_{t}^{c}}\right\|_{2} \leq d_{S S}
$$

where $\mathcal{X}^{\pi_{t}^{c}}$ is the 3D spatial location in Cartesian coordinate corresponding to the column index $\pi_{t}^{c}$ in matrix $\boldsymbol{\Delta} . \mathcal{X}^{\pi_{t}^{i}} \in$ $\mathcal{X}^{\pi_{t}} \cdot\|\cdot\|_{2}$ calculates the 2 -norms. $d_{S S}$ denotes spatial subspace radius and is a positive adjustable parameter.

Furthermore, we transform the set $\boldsymbol{d}_{t}^{\text {set, } x y z}$ from the 3D Cartesian coordinate to the vectorized column index set $\boldsymbol{d}_{t}^{\text {set }}$ (line 5 in Algorithm 2). For each column in $\boldsymbol{d}_{t}^{\text {set }}$, we solve the least squares problem and calculate the residual (line 6 to line 13 in Algorithm 2):

$$
\begin{gathered}
\hat{\boldsymbol{z}}_{\pi_{t}^{j}}^{S S}=\underset{\boldsymbol{z}_{\pi_{t}^{S S}}^{\arg \min }}{\arg }\left\|\boldsymbol{y}^{3 D S M}-\left(\boldsymbol{B} \cup\left\{\boldsymbol{\delta}_{\pi_{t}^{j}}\right\}\right) \cdot \boldsymbol{z}_{\pi_{t}^{j}}^{S S}\right\|_{2}, \\
\boldsymbol{q}_{\pi_{t}^{j}}^{S S}=\boldsymbol{y}^{3 D S M}-\left(\boldsymbol{B} \cup\left\{\boldsymbol{\delta}_{\pi_{t}^{j}}\right\}\right) \cdot \hat{\boldsymbol{z}}_{\pi_{t}^{j}}^{S S},
\end{gathered}
$$

where $\boldsymbol{B}$ represents all the optimally selected columns in $\boldsymbol{\Delta}$, $\delta_{\pi_{t}^{j}}$ is $j$-th column in the spatial subspace. $\hat{z}_{\pi_{t}^{j}}^{S S}$ is estimated signal power of signal sources when adding $\boldsymbol{\delta}_{\pi_{t}^{j}}$ into $\boldsymbol{B}$, and $\boldsymbol{q}_{\pi_{t}^{j}}^{S S}$ is the corresponding spatial subspace residual. The total residual set $\boldsymbol{q}_{\pi_{t}}^{S S}$ is thereby augmented with the new residual by

$$
\boldsymbol{q}_{\pi_{t}}^{S S}=\boldsymbol{q}_{\pi_{t}}^{S S} \cup\left\{\boldsymbol{q}_{\pi_{t}^{j}}^{S S}\right\} .
$$

At last, the corresponding index of the minimal residual in the spatial subspace can be obtained as follows (line 14 in
Algorithm 2 3D Spatial Subspace Based OMP Algorithm For 3D Compressed Spectrum Mapping Recovery

\section{Input:}

Initial residual vector $\boldsymbol{q}_{0}=\boldsymbol{y}^{3 D S M}$; Initial source location set $\boldsymbol{\Lambda}_{0}=\emptyset$; Sampling ratio $r=m / n$; Sensing matrix $\boldsymbol{\Delta}=\boldsymbol{\phi}^{3 D S M} \boldsymbol{\psi}^{3 D S M} \in \mathbb{R}^{m \times n} ;$ Iteration counter $t$; Initial chosen column set $\Gamma_{0}=\emptyset$; Iteration error threshold therror; Spatial subspace radius $d_{S S}$; Initial recovered sparse signal representation $\hat{s}=z \operatorname{eros}(n, 1)$;

\section{Output:}

The RMSE of the 3D recovered spectrum map $\mathcal{X}, W_{\mathcal{X}}$; Recovered sparse signal representation $\hat{s}$;

1: for $t=1 ; t \leq m ; t++$ do

2: $\quad$ Calculate $\pi_{t}^{c}$ by Eq. (22);

3: $\quad$ Transform $\pi_{t}^{c}$ to $\mathcal{X}^{\pi_{t}^{c}}$;

4: $\quad$ Find set $\boldsymbol{d}_{t}^{\text {set,xyz }}$ subject to Eq. (23);

5: Transform $\boldsymbol{d}_{t}^{\text {set,xyz }}$ to $\boldsymbol{d}_{t}^{\text {set }}$;

6: $\quad$ Set $\boldsymbol{q}_{\pi_{t}}^{S S}=\emptyset$;

7: $\quad$ for $j=1 ; j \leq \operatorname{length}\left(\boldsymbol{d}_{t}^{\text {set }}\right) ; j++$ do

8: $\quad$ Set $\Pi=\boldsymbol{\Lambda}_{t-1}, \boldsymbol{B}=\boldsymbol{\Gamma}_{t-1}$;

9: $\quad \boldsymbol{\Pi}=\boldsymbol{\Pi} \cup\left\{\pi_{t}^{j}\right\}, \boldsymbol{B}=\boldsymbol{B} \cup\left\{\boldsymbol{\delta}_{\pi_{t}^{j}}\right\} ;$

10: $\quad$ Solve Eq. (24) to obtain $\hat{z}_{\pi_{t}^{j}}^{S S}$;

11: $\quad \boldsymbol{q}_{\pi_{t}^{j}}^{S S}=\boldsymbol{y}^{3 D S M}-\boldsymbol{B} \hat{\boldsymbol{z}}_{\pi_{t}^{j}}^{S S}$

12: $\quad \boldsymbol{q}_{\pi_{t}}^{S S}=\boldsymbol{q}_{\pi_{t}}^{S S} \cup\left\{\boldsymbol{q}_{\pi_{t}^{j}}^{S S}\right\}$;

13: end for

14: $\quad$ Get $\pi_{t}^{\min }$ and $\boldsymbol{\delta}_{\pi_{t}^{\min }}$ as Eq. (27);

15: $\quad \boldsymbol{\Lambda}_{t}=\boldsymbol{\Lambda}_{t-1} \cup\left\{\pi_{t}^{\min }\right\}, \boldsymbol{\Gamma}_{t}=\boldsymbol{\Gamma}_{t-1} \cup\left\{\boldsymbol{\delta}_{\pi_{t}^{\min }}\right\} ;$

16: $\quad$ Solve $\hat{\boldsymbol{z}}_{t}=\arg \min \left\|\boldsymbol{y}^{3 D S M}-\boldsymbol{\Gamma}_{t} \boldsymbol{z}_{t}\right\|_{2}$;

17: $\quad$ Get residual $\boldsymbol{q}_{t}=\boldsymbol{y}^{3 D S M}-\boldsymbol{\Gamma}_{t} \hat{\boldsymbol{z}}_{t}$;

18: $\quad$ Set $\boldsymbol{\Delta}_{\pi_{\text {min }}}=\operatorname{zeros}(m, 1)$;

19: $\quad$ if $\left|\hat{\boldsymbol{z}}_{t}(t)\right| /\left\|\hat{\boldsymbol{z}}_{t}\right\|_{2}<t h_{\text {error }}$ then

20: $\quad$ Break

21: end if

22: end for

23: Obtain $\boldsymbol{\Lambda}, \hat{z}$, and $\hat{\boldsymbol{s}}(\boldsymbol{\Lambda})=\hat{\boldsymbol{z}}, \hat{\boldsymbol{x}}_{\mathcal{X}}=\boldsymbol{\psi}^{3 D S M} \hat{\boldsymbol{s}}$;

24: Output $W_{\mathcal{X}}$ and $\hat{s}$.

\section{Algorithm 2):}

$$
\pi_{t}^{\min }=\underset{\pi_{t}^{i}}{\arg \min }\left\|\boldsymbol{q}_{\pi_{t}^{i}}^{S S}\right\|_{2} .
$$

Subsequently, the estimated signal power vector $\hat{z}_{t}$ and the residual $\boldsymbol{q}_{t}$ corresponding to $\pi_{t}^{\min }$ in the $t$-th iteration are obtained (line 15 to 17 in Algorithm 2), the selected column is also set to zero (line 18 in Algorithm 2).

After $m$ iterations of the outer for loop, or when the successive iteration deviation of $\hat{z}_{t}$ is less than the threshold $t h_{\text {error }}$, we get the final estimated signal source locations $\boldsymbol{\Lambda}$ and their transmission powers $\hat{z}$. Upon $\hat{s}(\boldsymbol{\Lambda})=\hat{z}$, the entire situation can be obtained as $\hat{x}_{\mathcal{X}}=\boldsymbol{\psi}^{3 D S M} \hat{\boldsymbol{s}}$.

The time complexity of the proposed 3D spatial subspace based OMP algorithm focuses on the maximum column projection calculation in line 2, the 3D spatial subspace minimal residual iterations from line 7 to line 13 and the iterative signal estimation operation in line 16 . The column projec- 


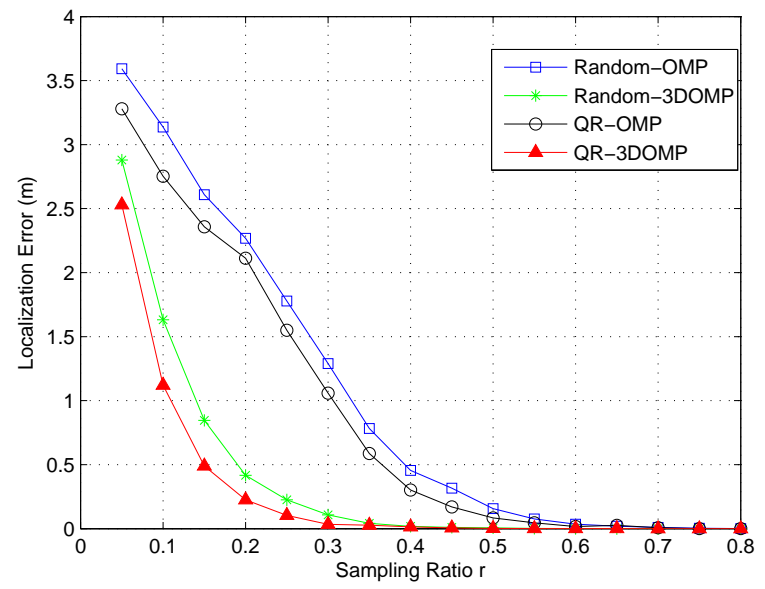

Fig. 6. Signal sources localization performance comparisons of RandomOMP, Random-3DOMP, QR-OMP and QR-3DOMP $\left(d_{S S}=3\right)$.

tion calculation is a simple process with computational cost $\mathcal{O}(m n)$. The least squares problem solution in line 10 has a time complexity of $\mathcal{O}\left(j^{3}\right)$. The cost of residual calculation in line 11 is $\mathcal{O}(m j)$. Similarly, the time complexity of line 16 is $\mathcal{O}\left(t^{3}+m t\right)$. Therefore, the complexity summation of $3 \mathrm{D}$ spatial subspace minimal residual iterations can be obtained as $\mathcal{O}\left(\sum_{j=1}^{\left|\boldsymbol{d}_{t}^{\text {set }}\right|}\left(j^{3}+m j\right)\right) \approx \mathcal{O}\left(\left|\boldsymbol{d}_{t}^{\text {set }}\right|^{3}+m \cdot\left|\boldsymbol{d}_{t}^{\text {set }}\right|\right)$. In general, after $m$ outer for loops, the total computational cost $\mathcal{C}_{2}$ is obtained as

$$
\begin{aligned}
\mathcal{C}_{2} & =O\left(m \cdot(m n)+m\left(\left|d_{t}^{\text {set }}\right|^{3}+m\left|d_{t}^{\text {set }}\right|\right)+\sum_{t=1}^{m}\left(t^{3}+m t\right)\right) \\
& \approx O\left(m^{2} n+m\left|d_{t}^{\text {set }}\right|^{3}+m^{2}\left|d_{t}^{\text {set }}\right|+m^{3}+m^{2}\right) \\
& \approx O\left(m^{2} n+m^{3}\right),
\end{aligned}
$$

where $\left|\boldsymbol{d}_{t}^{\text {set }}\right|$ is a constant of 3D spatial subspace size and is usually small compared to sampling number $m$ and original signal length $n$.

\section{EXPERIMENTAL RESULTS AND DISCUSSIONS}

\section{A. Experiment Setup}

In this section, the performance of the proposed 3D spectrum mapping scheme is evaluated through simulations. The parameters are set as follows: First, we consider a 3D city block with a size of $100 \mathrm{~m} \times 100 \mathrm{~m} \times 100 \mathrm{~m}$. We take a spatial granularity of $10 \mathrm{~m}$ and discretize the space into a spectrum tensor of $N_{1} \times N_{2} \times N_{3}=10 \times 10 \times 10$. The source number is 5 . The signal power of the source is assumed to be $30 \mathrm{~mW}$. 3D spatial subspace based OMP algorithm iteration threshold therror $=5 \times 10^{-4}$. The receiver noise spectral density is $-174 \mathrm{dBm} / \mathrm{Hz}$ and the bandwidth is $200 \mathrm{KHz}$.

\section{B. Comparison of Localization Performance}

First, as shown in Fig. 6, the relationship between the localization error of 3D sparse spatial signal sources and the

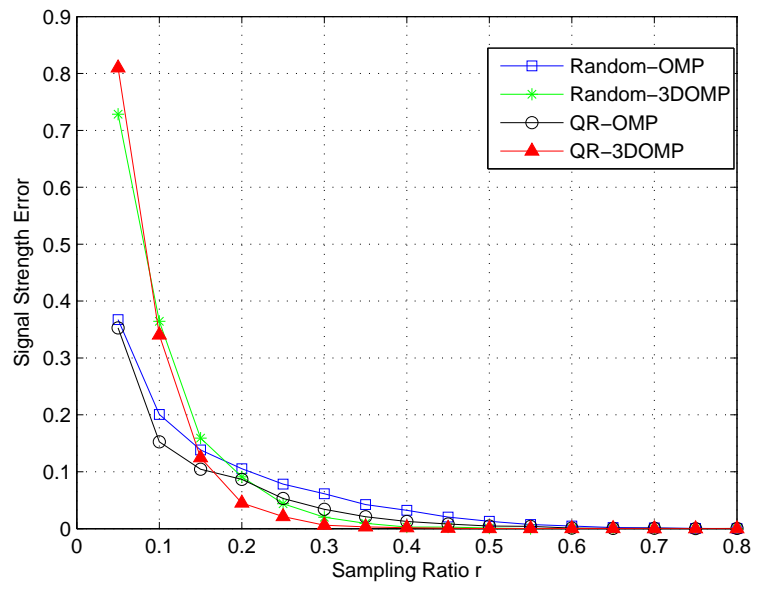

Fig. 7. Source signal strength recovery performance comparisons of RandomOMP, Random-3DOMP, QR-OMP and QR-3DOMP $\left(d_{S S}=3\right)$.

sampling ratio is studied. The average localization error is defined as:

$$
\text { localization_error }=\frac{\sum_{i=1}^{k} \| \operatorname{location}^{(i)}-\text { truelocation }^{(i)} \|_{2}}{k},
$$

where location ${ }^{(i)}$ and truelocation ${ }^{(i)}$ represent estimated and true signal sources locations in the Cartesian coordinate. $k$ is the sparsity of signal which is the number of signal sources in the 3D space.

Four different algorithms are compared, including RandomOMP, Random-3DOMP, QR-OMP and QR-3DOMP. Given sampling ratio $r$, "Random" means sampling locations are arbitrarily selected from the 3D space. "QR" means Algorithm 1 in Section IV.A is adopted to optimize the location selection. "OMP" is a recovery method used in traditional compressed sensing [27]. "3DOMP" is our proposed Algorithm 2 mentioned in Section IV.B. It can be seen from the simulation results that: $i$ ) For all algorithms, localization error decreases as the number of sampling points increases; ii) Our proposed 3D spectrum mapping scheme outperforms the others; iii) In addition, Random-3DOMP and QR-3DOMP algorithms are much better than the RandomOMP and QR-OMP algorithm on the localization accuracy. It reveals that the selected locations through $\mathrm{QR}$ pivoting based sampling optimization can better guarantee sparse recovery than random sampling. In addition, 3D spatial subspace based OMP algorithm brings more performance improvement than QR sampling optimization in signal source location estimation. Through spatial subspace search, the accuracy of signal source localization is further improved by nearly $2 \mathrm{~m}$ when $r=0.2$.

\section{Comparison of Signal Strength Recovery Performance}

Here we compare the source signal strength recovery performance among the four cases Random-OMP, Random3DOMP, QR-OMP and QR-3DOMP. The average relative 


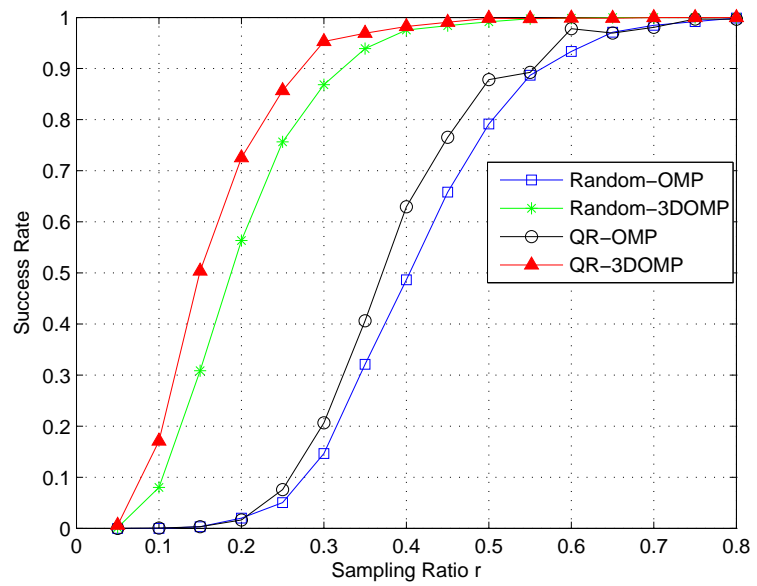

Fig. 8. Recovery success ratio comparisons of Random-OMP, Random3DOMP, QR-OMP and QR-3DOMP $\left(d_{S S}=3\right)$.

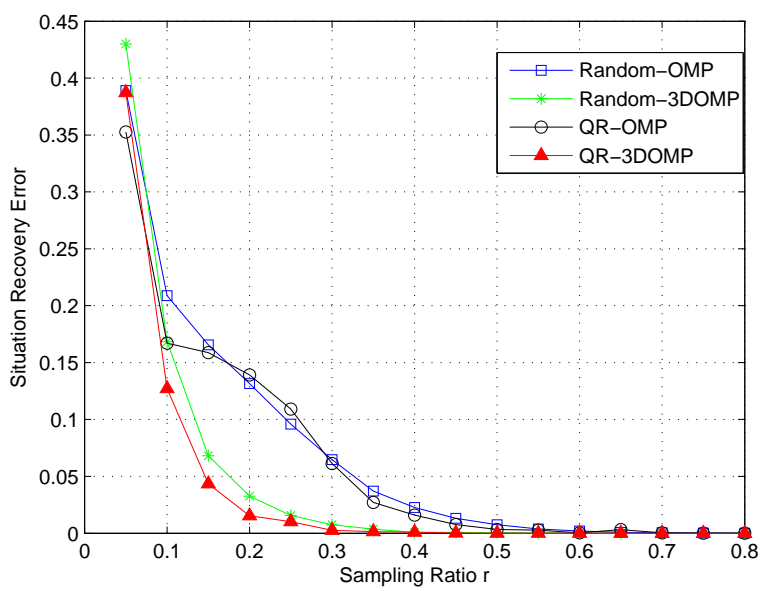

Fig. 9. 3D spectrum situation recovery performance comparisons of RandomOMP, Random-3DOMP, QR-OMP and QR-3DOMP $\left(d_{S S}=3\right)$.

signal strength recovery error is

signalstrength_error $=\frac{1}{k} \sum_{i=1}^{k}\left|\frac{\text { signal }^{(i)}-\operatorname{truesignal}^{(i)}}{\operatorname{truesignal}^{(i)}}\right|^{2}$,

where signal $^{(i)}$ and truesignal ${ }^{(i)}$ denote estimated and true sources signal strengthes, respectively. Results are shown in Fig. 7: when the sampling ratio is less than 0.15 , the Random and $\mathrm{QR}$ algorithms with OMP have lower errors than the 3DOMP algorithm. But when sampling ratio exceeds 0.15 , the performances of Random-3DOMP and QR-3DOMP are better. It can be concluded that when the sampling ratio is very low, although 3DOMP algorithm brings more accurate signal source locations, the source signal strength recovery is relatively inferior. This indicates 3DOMP is more sensitive to localization errors which cause more signal strength recovery errors than OMP.
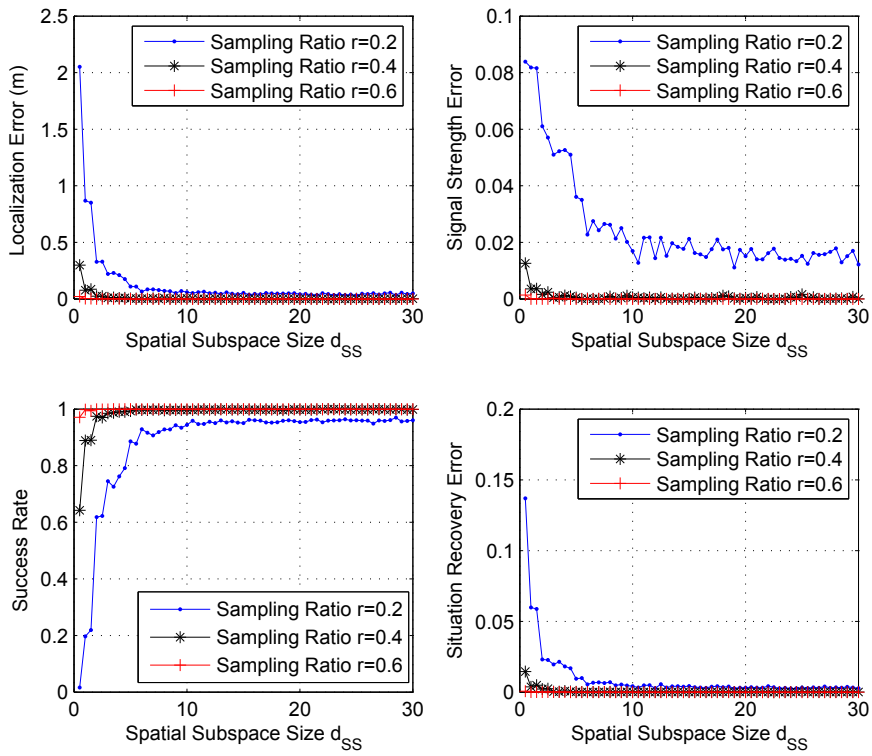

Fig. 10. Impact of spatial subspace size $d_{S S}$ on the QR-3DOMP performances of localizations, signal strength recovery, success rate and spectrum situation recovery $(r=0.2,0.4,0.6)$.

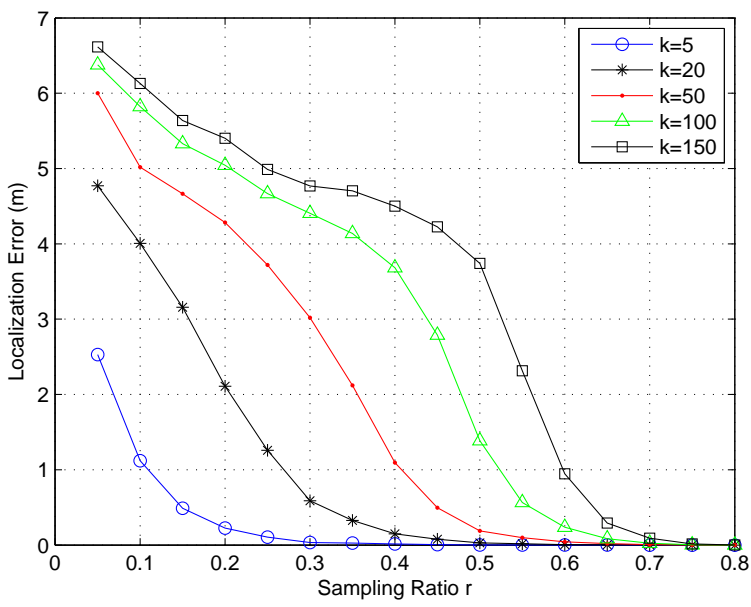

Fig. 11. Impact of sparsity $k$ on the QR-3DOMP performances of localizations $\left(d_{S S}=3\right)$.

\section{Comparison of Recovery Success Rate}

In Fig. 8, we study the recovery success ratio of four algorithms, which is defined as:

$$
\begin{aligned}
\text { success_ratio }= & \frac{\mid\left\{i \text { :success_error }(i)<\tau, 1 \leq i \leq N_{\text {Monte }}\right\} \mid}{N_{\text {Monte }}}, \\
& \text { success_error }=\|\hat{\boldsymbol{s}}-\boldsymbol{s}\|_{2},
\end{aligned}
$$

where $\hat{s}$ and $s$ represent the estimated and original sparse signals. $N_{\text {Monte }}$ is the total number of Monte Carlo process. $\tau$ denotes the error threshold to decide whether it is a successful sparse signal recovery $\left(N_{\text {Monte }}=5000, \tau=0.1\right.$ in our simulation). $|\cdot|$ returns the element number of a set.

It can be seen that the performances of Random-3DOMP and QR-3DOMP are much better than the other two algorithms. The success rates of Random-3DOMP and QR-3DOMP are 


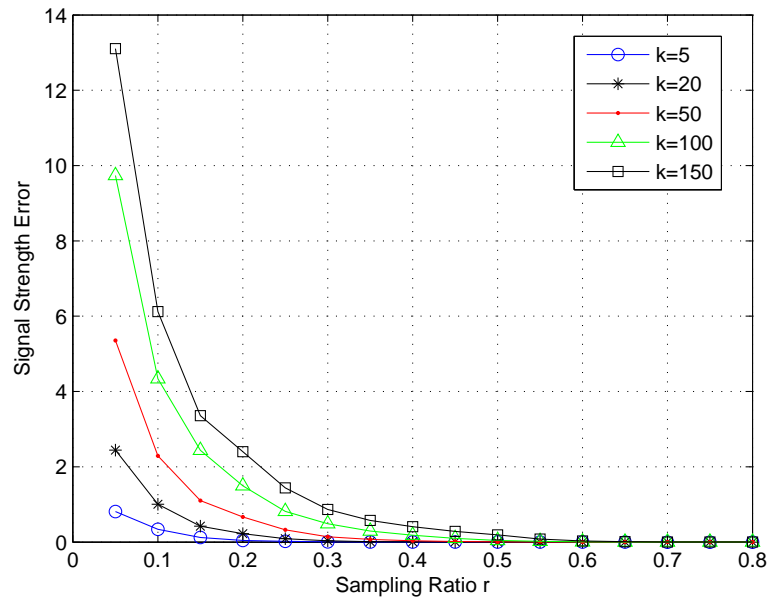

Fig. 12. Impact of sparsity $k$ on the QR-3DOMP performances of signal strength recovery $\left(d_{S S}=3\right)$.

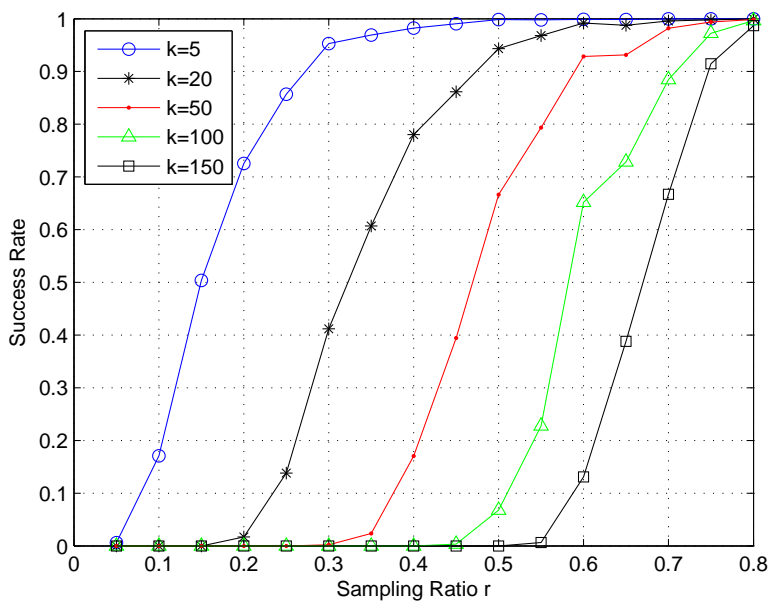

Fig. 13. Impact of sparsity $k$ on the QR-3DOMP performances of success rate $\left(d_{S S}=3\right)$.

close to 1 when the sampling ratio is about 0.4. However, the Random-OMP and QR-OMP require a sampling ratio of 0.7 to achieve the same performance. What's more, the sparse signal recovery errors of OMP algorithm are always larger than the threshold $\tau$ when the sampling ratio is less than 0.15. Compared with Fig. 7 , we can get that the nonzero elements number of the OMP recovered sparse signal is much larger than sparsity $k$. Generally speaking, the proposed 3D spectrum mapping scheme can greatly increase the probability of successful recovery.

\section{E. Comparison of Situation Recovery Performance}

Moreover, the 3D spectrum situation recovery performance is studied as showed in Fig. 9. It calculates the average relative situation recovery error of a single spatial point as below:

situation_error $=\frac{1}{n} \sum_{i=1}^{n}\left|\frac{\text { situation }^{(i)}-\text { truesituation }^{(i)}}{\text { truesituation }^{(i)}}\right|^{2}$,

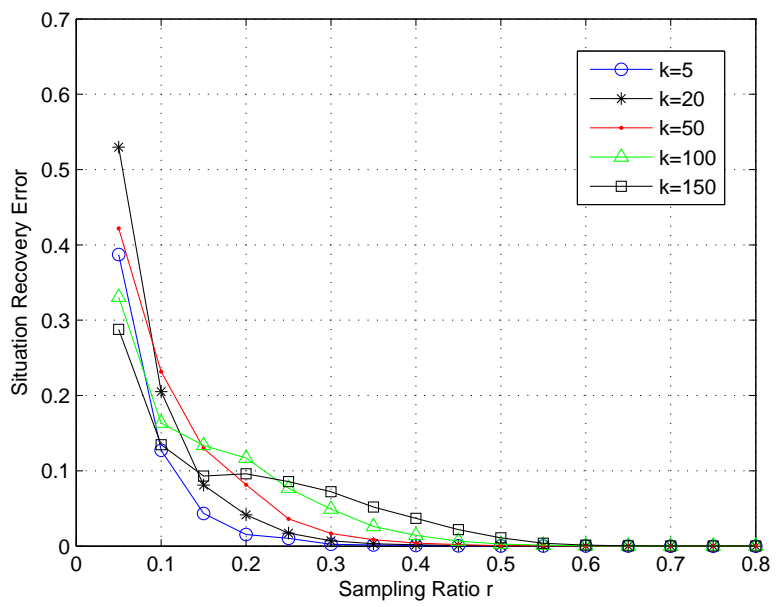

Fig. 14. Impact of sparsity $k$ on the QR-3DOMP performances of spectrum situation recovery $\left(d_{S S}=3\right)$.

where situation $^{(i)}$ and truesituation ${ }^{(i)}$ denote estimated and true spatial point situation values, respectively. Generally, our proposed QR-3DOMP algorithm is outstanding in situation recovery. In comparison with Fig. 6, 7, and 9, it can be seen that when the sampling rate is lower than 0.1 , the performance of QR-3DOMP is close to the others. But when it's larger than 0.1, the two 3DOMP based algorithms outperform the others by a huge advantage. This is a result of both superior performance in signal source localization and sparse signal recovery.

\section{F. Impact of Spatial Subspace Size $d_{S S}$}

Furthermore, we study the impact of the 3D spatial subspace size $d_{S S}$ on the performance of the proposed QR-3DOMP algorithm, as shown in Fig. 10. It can be found that the performances including localizations, signal strength recovery, success rate and spectrum situation recovery are all improved and then gradually converge as $d_{S S}$ increases. Meanwhile, a large $d_{S S}$ also brings more computations as more column candidates exist in the spatial subspace. In addition, when the sampling rate $r$ grows, all performances obtain great improvements. When $r$ reaches 0.6 , the success rate is almost 1 , and the recovery errors are close to 0 . These observations indicate that appropriate increasement of spatial subspace radius can reduce an amount of sampling.

\section{G. Impact of Sparsity $k$}

The sparsity $k$ is an important parameter for sparse recovery in the compressed sensing theory. The effects of $k$ on the proposed QR-3DOMP algorithm are shown in Fig. 11 to 14. It can be seen from Fig. 11 and Fig. 12 that as the sparsity $k$ increases, the localization error and the signal strength recovery error both increase. Fig. 13 shows that the success rate of signal recovery decreases as $k$ increases, and approximately when $m>2 k \ln (n / k)$, it is possible to successfully recover the signal. In addition, for spectrum situation recovery in Fig. 14: when the sampling ratio $r$ is small, the situation recovery 


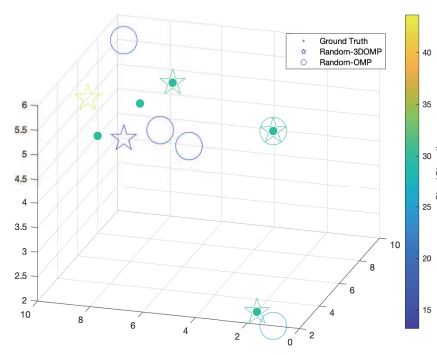

(a) Localization and signal strength recovery (Random-0MP and Random-3DOMP)

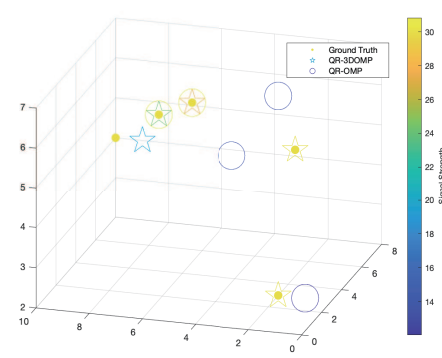

(b) Localization and signal strength recovery (QR-OMP and QR-3DOMP)

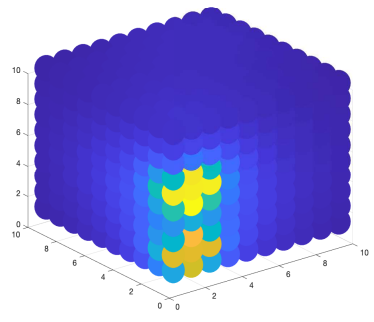

(c) Random-0MP

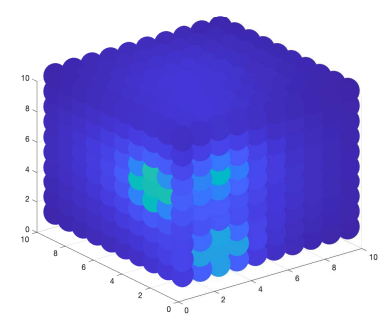

(d) QR-OMP

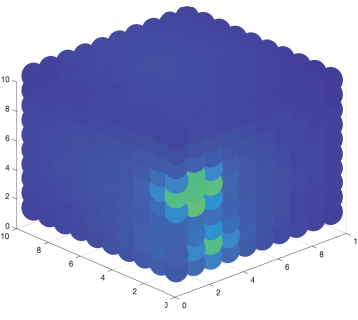

(e) Random-3DOMP

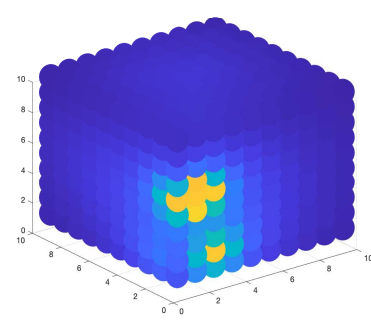

(f) QR-3DOMP

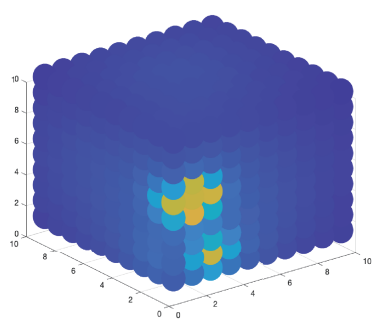

(g) Ground Truth

Fig. 15. 3D spectrum mapping performance visualization ( $r=0.1, d_{S S}=3$ ). (a) and (b) present the localizations and signal strength recovery of RandomOMP, Random-3DOMP and QR-OMP, QR-3DOMP, respectively. (c), (d), (e) and (f) show the spectrum situation recovery. (g) is the ground truth of spectrum situation.

error first increases and then decreases with the increasing $k$; When $r$ is large, the situation recovery error monotonously increases with $k$.

\section{H. 3D Spectrum Mapping Performance Visualization}

At last, performance visualizations are made in Fig. 15 to intuitively present the recovery effects of the above RandomOMP, Random-3DOMP, QR-OMP and QR-3DOMP algorithms on localization, source signal strength and 3D spectrum situation. In Fig. 15(a) and (b) on the left, the color bar represents different signal strengths. The solid dot, pentagon, and circle represent the ground truth and the recovered signal source location of four algorithms. If the pentagon or circle overlaps with the solid dot, it means that one of the recovered signal source is accurately located. The comparison of (c)$(\mathrm{g})$ on the right further proves our proposed mapping scheme performs well in signal source localization and source signal strength recovery visually.

\section{CONCLUSION}

In this paper, we have investigated the issue of 3D compressed spectrum mapping in spectrum-heterogeneous environment, which offers high value for efficient dynamic spectrum access of various RF devices. We first formulated 3D compressed spectrum mapping as a compressed sensing optimization problem by exploiting the underlying sparse nature of 3D spectrum situation. Then, we proposed a 3D compressed spectrum mapping scheme, which is composed of two components: sampling locations optimization and spectrum situation recovery. The former adopts QR pivoting optimization to determine dominant sampling locations and shows better performance than random measurement. Due to the ineffectiveness of traditional OMP recovery algorithm in 3D compressed spectrum mapping, a tailored 3D spatial subspace based OMP algorithm was proposed. Furthermore, we compared the localization, source signal strength recovery, recovery success rate and situation recovery performances among four schemes Random-OMP, Random-3DOMP, QROMP and QR-3DOMP. The impact of spatial subspace size and sparsity on situation recovery precision were also studied. Results show the superiority of the proposed 3D compressed spectrum mapping scheme. Further research will be conducted to investigate several issues including data-driven sparse dictionary learning algorithms to grab the signal transmission mechanism of practical scenarios and constraints of spectrum mapping, e.g., irregular surveillance area and energy consumption of spectrum-monitoring devices.

\section{REFERENCES}

[1] Z. Feng, C. Qiu, Z. Feng, Z. Wei, W. Li, and P. Zhang, "An effective approach to 5G: Wireless network virtualization," IEEE Communications Magazine, vol. 53, no. 12, pp. 53-59, Dec. 2015.

[2] Q. Wu, G. Ding, J. Wang, and Y. Yao, "Spatial-temporal opportunity detection for spectrum-heterogeneous cognitive radio networks: Twodimensional sensing," IEEE Transactions on Wireless Communications, vol. 12, no. 2, pp. 516-526, Feb. 2013.

[3] S. Stotas and A. Nallanathan, "On the throughput and spectrum sensing enhancement of opportunistic spectrum access cognitive radio networks," IEEE Transactions on Wireless Communications, vol. 11, no. 1, pp. 97-107, Jan. 2012.

[4] FCC, "Spectrum policy task force report," ET Docket 02-135, Nov. 2002

[5] — , "Facilitating opportunities for flexible, efficient, and reliable spectrum use employing cognitive radio technologies," ET Docket 03108, Dec. 2003.

[6] S. Haykin, "Cognitive radio: Brain-empowered wireless communications," IEEE Journal on Selected Areas in Communications, vol. 23, no. 2, pp. 201-220, Feb. 2005. 
[7] J. Mitola and G. Q. Maguire, "Cognitive radio: Making software radios more personal," IEEE Personal Communications, vol. 6, no. 4, pp. 1318, Aug. 1999.

[8] B. Wang and K. R. Liu, "Advances in cognitive radio networks: A survey," IEEE Journal of Selected Topics in Signal Processing, vol. 5, no. 1, pp. 5-23, Feb. 2011.

[9] Y. Chen, G. Yu, Z. Zhang, H.-h. Chen, and P. Qiu, "On cognitive radio networks with opportunistic power control strategies in fading channels," IEEE Transactions on Wireless Communications, vol. 7, no. 7, pp. 2752 2761, Jul. 2008

[10] Q. Zhao and B. M. Sadler, "A survey of dynamic spectrum access," IEEE Signal Processing Magazine, vol. 24, no. 3, pp. 79-89, May 2007.

[11] A. Goldsmith, S. A. Jafar, I. Maric, and S. Srinivasa, "Breaking spectrum gridlock with cognitive radios: An information theoretic perspective," Proceedings of the IEEE, vol. 97, no. 5, pp. 894-914, 2009.

[12] J. Schuette, B. Fell, J. Chapin, S. Jones, J. Stutler, M. Birchler, and D. Roberson, "Performance of RF mapping using opportunistic distributed devices," in 2015 IEEE Military Communications Conference, Tampa, FL, USA, Oct. 2015, pp. 1624-1629.

[13] G. Ding, J. Wang, Q. Wu, Y.-D. Yao, F. Song, and T. A. Tsiftsis, "Cellular-base-station-assisted device-to-device communications in TV white space," IEEE Journal on Selected Areas in Communications, vol. 34, no. 1, pp. 107-121, Jan. 2016.

[14] V. Atanasovski, J. van de Beek, A. Dejonghe, D. Denkovski, L. Gavrilovska, S. Grimoud, P. Mähönen, M. Pavloski, V. Rakovic, J. Riihijarvi et al., "Constructing radio environment maps with heterogeneous spectrum sensors," in 2011 IEEE International Symposium on Dynamic Spectrum Access Networks (DySPAN), Aachen, Germany, May 2011, pp. 660-661.

[15] G. Ding, Q. Wu, Y. Yao, J. Wang, and Y. Chen, "Kernel-based learning for statistical signal processing in cognitive radio networks: Theoretical foundations, example applications, and future directions," IEEE Signal Processing Magazine, vol. 30, no. 4, pp. 126-136, Jul. 2013.

[16] D. Romero, S. Kim, G. B. Giannakis, and R. Lpez-Valcarce, "Learning power spectrum maps from quantized power measurements," IEEE Transactions on Signal Processing, vol. 65, no. 10, pp. 2547-2560, May 2017.

[17] X. Han, L. Xue, F. Shao, and Y. Xu, "A power spectrum maps estimation algorithm based on generative adversarial networks for underlay cognitive radio networks," Sensors, vol. 20, p. 311, Jan. 2020.

[18] F. Shen, G. Ding, Z. Wang, and Q. Wu, "UAV-based 3D spectrum sensing in spectrum-heterogeneous networks," IEEE Transactions on Vehicular Technology, vol. 68, no. 6, pp. 5711-5722, Jun. 2019.

[19] M. Tang, G. Ding, Q. Wu, Z. Xue, and T. A. Tsiftsis, "A joint tensor completion and prediction scheme for multi-dimensional spectrum map construction," IEEE Access, vol. 4, pp. 8044-8052, Nov. 2016.

[20] J. Sun, J. Wang, G. Ding, L. Shen, J. Yang, Q. Wu, and L. Yu, "Longterm spectrum state prediction: An image inference perspective," IEEE Access, vol. 6, pp. 43 489-43 498, Jul. 2018.

[21] C. Ge, Z. Wang, and X. Zhang, "Robust long-term spectrum prediction with missing values and sparse anomalies," IEEE Access, vol. 7, pp 16655-16664, Jan. 2019.

[22] D. L. Donoho, "Compressed sensing," IEEE Transactions on Information Theory, vol. 52, no. 4, pp. 1289-1306, May 2006.

[23] E. Candes and T. Tao, "Near-optimal signal recovery from random projections: Universal encoding strategies?" IEEE Transactions on Information Theory, vol. 52, pp. 5406 - 5425, Jan. 2007.

[24] M. Elad, "Optimized projections for compressed sensing," IEEE Transactions on Signal Processing, vol. 55, pp. 5695 - 5702, Jan. 2008.

[25] E. Candes, J. Romberg, and T. Tao, "Robust uncertainty principles: exact signal reconstruction from highly incomplete frequency information," IEEE Transactions on Information Theory, vol. 52, no. 2, pp. 489-509, Feb. 2006.

[26] S. G. Mallat and Zhifeng Zhang, "Matching pursuits with time-frequency dictionaries," IEEE Transactions on Signal Processing, vol. 41, no. 12, pp. 3397-3415, Dec. 1993.

[27] J. A. Tropp and A. C. Gilbert, "Signal recovery from random measurements via orthogonal matching pursuit," IEEE Transactions on Information Theory, vol. 53, no. 12, pp. 4655-4666, Dec. 2007.

[28] Z. Wang and C. Ling, "On the geometric ergodicity of MetropolisHastings algorithms for lattice Gaussian sampling," IEEE Transactions on Information Theory, vol. 64, no. 2, pp. 738-751, Feb. 2018.

[29] _ - "Lattice Gaussian sampling by Markov chain Monte Carlo: Bounded distance decoding and trapdoor sampling," IEEE Transactions on Information Theory, vol. 65, no. 6, pp. 3630-3645, June 2019.
[30] K. Manohar, B. W. Brunton, J. N. Kutz, and S. L. Brunton, "Data-driven sparse sensor placement for reconstruction: Demonstrating the benefits of exploiting known patterns," IEEE Control Systems Magazine, vol. 38, no. 3, pp. 63-86, Jun. 2018.

[31] Q. Wu, F. Shen, Z. Wang, and G. Ding, "3D spectrum mapping based on ROI-driven UAV deployment," IEEE Network, vol. 34, no. 5, pp. $24-31,2020$.

[32] T. F. Chan, "Rank revealing QR factorizations," in Linear Algebra Appl., vol. 88-89, Apr. 1987, pp. 67-82.

[33] J. A. Duersch and M. Gu, "Randomized QR with column pivoting," SIAM Journal on Scientific Computing, vol. 39, no. 4, pp. C263-C291, Jan. 2017.

[34] T. F. Chan and P. C. Hansen, "Some applications of the rank revealing QR factorization," SIAM Journal on Scientific and Statistical Computing, vol. 13, no. 3, pp. 727-741, May 1992.

[35] P. Businger and G. H. Golub, "Linear least squares solutions by Householder transformations," Numerische Mathematik, vol. 7, no. 3, pp. 269-276, Jun. 1965.

[36] A. Sommariva and M. Vianello, "Computing approximate Fekete points by QR factorizations of Vandermonde matrices," Computers \& Mathematics with Applications, vol. 57, no. 8, pp. 1324-1336, Apr. 2009.

[37] Z. Drmac and S. Gugercin, "A new selection operator for the discrete empirical interpolation method-improved a priori error bound and extensions," SIAM Journal on Scientific Computing, vol. 38, no. 2, pp. A631-A648, May 2016. 


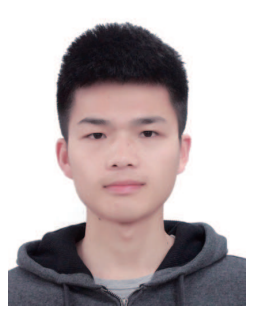

Feng Shen received his B.S. degree in information engineering from Nanjing University of Aeronautics and Astronautics, Nanjing, China, in 2017. He is currently pursuing his Ph.D. degree at the College of Electronics and Information Engineering, Nanjing University of Aeronautics and Astronautics, Nanjing, China. His research interests include cognitive information theory, cognitive radio, signal processing and wireless communications.

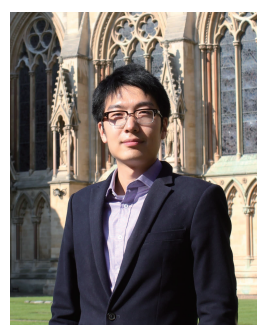

Kezhi Li received his B.Eng. degree at University of Science and Technology of China (USTC) in 2008, and the Ph.D. degree at Imperial College London (ICL) in 2012. He is now a lecturer at Institute of Health Informatics (IHI), University College London (UCL). Prior to joining UCL, he was a senior research associate at ICL, University of Cambridge, a research fellow at Royal Institute of Technology $(\mathrm{KTH})$ in Stockholm and a research assistant at Microsoft Research Asia (MSRA). His research interests are quite broad in several inter-discipline areas, such as biomedical signal processing/time series analysis in glucose management, patient flow optimization, critical care condition prediction, using machine learning, data mining and computer vision, structured signal processing and their applications in imaging system, quantum tomography and information theory.

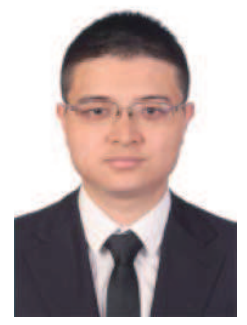

Zheng Wang received the B.S. degree in electronic and information engineering from Nanjing University of Aeronautics and Astronautics, Nanjing, China, in 2009, and the M.S. degree in communications from the Department of Electrical and Electronic Engineering, University of Manchester, Manchester, U.K., in 2010. He received the Ph.D. degree in communication engineering from Imperial College London, UK, in 2015.

From 2015 to 2016 he served as a Research Associate at Imperial College London, UK and from 2016 to 2017 he was an senior engineer with Radio Access Network R\&D division, Huawei Technologies Co.. From 2017 to 2021, he served as a Lecturer \& Associate Professor at the College of Electronics and Information Engineering, Nanjing University of Aeronautics and Astronautics (NUAA), Nanjing, China. He is currently an Associate Professor at the School of Information Science and Engineering, Southeast University, Nanjing, China. His current research interests include lattice methods for wireless communications, machine learning and intelligent technology for communications.

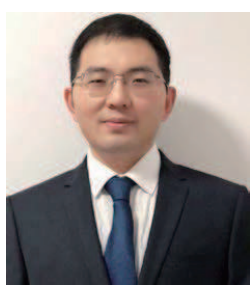

Guoru Ding (''10-M'14-SM'16) is currently an Associate Professor with the College of Communications Engineering, Nanjing, China. He received the B.S. (Hons.) degree in electrical engineering from Xidian University, Xi' an, China, in 2008, and the $\mathrm{Ph} . \mathrm{D}$. (Hons.) degree in communications and information systems from the College of Communications Engineering, Nanjing, China, in 2014. From 2015 to 2018, he was a Post-Doctoral Research Associate with the National Mobile Communications Research Laboratory, Southeast University, Nanjing, China. His research interests include cognitive radio networks, massive MIMO, machine learning and data analytics over wireless networks.

He has received the Excellent Doctoral Thesis Award of the China Institute of Communications in 2016, the Alexander von Humboldt Fellowship in 2017, the Excellent Young Scientist of Wuwenjun Artificial Intelligence in 2018 , and the 14th IEEE COMSOC Aisa-Pacific Outstanding Young Researcher Award in 2019. He was a recipient of the Natural Science Foundation for Distinguished Young Scholars of Jiangsu Province, China and six best paper awards from international conferences such as the IEEE VTC-FALL 2014. He has served as a Guest Editor for the IEEE JOURNAL ON SELECTED AREAS IN COMMUNICATIONS (special issue on spectrum sharing and aggregation in future wireless networks). He is currently an Associate Editor of the IEEE TRANSACTIONS ON COGNITIVE COMMUNICATIONS AND NETWORKING and a Technical Editor of the IEEE 1900.6 Standard Association Working Group.

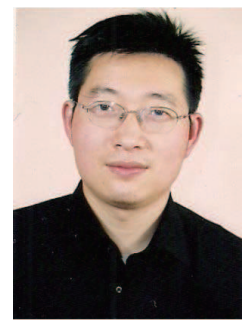

Qihui Wu (SM'13) received his B.S. degree in communications engineering, M.S. degree and Ph.D. degree in communications and information systems from Institute of Communications Engineering, Nanjing, China, in 1994, 1997 and 2000, respectively. From 2003 to 2005, he was a Postdoctoral Research Associate at Southeast University, Nanjing, China. From 2005 to 2007, he was an Associate Professor with the College of Communications Engineering, PLA University of Science and Technology, Nanjing, China, where he served as a Full Professor from 2008 to 2016. Since May 2016, he has been a full professor with the College of Electronic and Information Engineering, Nanjing University of Aeronautics and Astronautics, Nanjing, China. From March 2011 to September 2011, he was an Advanced Visiting Scholar in Stevens Institute of Technology, Hoboken, USA. Dr. Wu's current research interests span the areas of wireless communications and statistical signal processing, with emphasis on system design of software defined radio, cognitive radio, and smart radio. 